\title{
Perspective of dairy producers from California, Idaho, South Dakota, and Washington: Health and business implications of the COVID-19 pandemic during the second wave
}

\author{
A. Valldecabres, ${ }^{1,2} \odot$ J. Wenz, ${ }^{3}$ F. C. Ferreira, ${ }^{1,2} \odot$ M. Chahine, ${ }^{4} \odot$ J. Dalton, ${ }^{5} \odot$ M. de Haro Marti, ${ }^{6} \odot$ M. Rovai, ${ }^{7} \odot$ \\ and N. Silva-del-Río ${ }^{1,2 *}$ (1) \\ ${ }^{1}$ Veterinary Medicine Teaching and Research Center, 18830 Road 112, Tulare, CA 93274 \\ ${ }^{2}$ Department of Population Health and Reproduction, School of Veterinary Medicine, University of California, Davis 95616 \\ ${ }^{3}$ Washington State University, Pullman 99164 \\ ${ }^{4}$ University of Idaho, Twin Falls Research and Extension Center, Twin Falls 83301 \\ ${ }^{5}$ University of Idaho, Caldwell Research and Extension Center, Caldwell 83605 \\ ${ }^{6}$ University of Idaho, Extension Gooding County, Gooding 83330 \\ ${ }^{7}$ Dairy and Food Science Department, South Dakota State University, Brookings 57007
}

\begin{abstract}
The aim of this study was to document California, Idaho, South Dakota, and Washington dairy producers' perspectives on the health and business implications of COVID-19 during the pandemic second wave. Dairy producers were reached by a 14-question anonymous mail survey during June and July of 2020. We obtained 226 responses (response rate: $9.3 \%$ CA, $8.6 \%$ ID, $31.4 \%$, and $10.0 \% \mathrm{WA}$ ). Responses were grouped by state (CA: 48.7\%, ID: $15.9 \%$, SD: $21.7 \%$, or WA: $13.7 \%)$ and dairy size [based on number of cows; small $(<100)$ : $14.1 \%$, medium (100-499): 27.7\%, medium-large (500-1,999): $33.2 \%$, or large ( $\geq 2,000): 25.0 \%]$. Survey responses were summarized, and multiple correspondence analysis was used to map responses and identify data clusters. At the time of the survey, some respondents suspected $(3 \%)$ or had confirmed (9\%) COVID-19 cases on their premises. Respondents were somewhat or very concerned about the health $(75 \%)$ and business (92\%) implications associated with COVID-19. Producers were concerned about the health of their families, employees, and employees' families; having to reduce production; workforce shortage; limited goods availability; and lack of services. Producers perceived that their employees were somewhat or very concerned (74\%) with the pandemic. There was COVID-19 safety information or training provided $(78 \%)$ or intended (4\%) in English $(22 \%)$, Spanish (23\%), or both (55\%). The focus of training was as follows: how to remain healthy at work (91\%) and at home $(60 \%)$, what to do if a worker gets sick (77\%), and sick leave information (54\%). Nevertheless,
\end{abstract}

Received June 25, 2021.

Accepted October 28, 2021.

*Corresponding author: nsilvadelrio@ucdavis.edu
$18 \%$ of the respondents answered that training was not going to be provided. The following control measures implemented: providing hand sanitizer or disinfectant wipes $(78 \%)$, instruction on frequent hand-washing (76\%), social distancing (59\%), prevention of employee gatherings (54\%), providing and requiring the use of face masks (49\%), and limiting on-farm visitors (44\%). Multiple correspondence analysis showed that concern with health risk and business impact of the COVID-19 pandemic, as well as mitigation and response strategies implemented, varied based on respondent's dairy size and state. The greatest concerns were reported by producers from California and large and medium-large dairies, whereas the fewest concerns were reported by producers from small dairies. Results from this survey highlighted the health and business concerns of dairy producers from California, Idaho, South Dakota, and Washington during the COVID-19 pandemic and the mitigation efforts adopted. In conclusion, mitigation measures were adopted by most dairy producers but in various degrees. Dairy industry service providers and educators were secondary educational resources during the pandemic; thus, future efforts toward centralized access to dairy specific bilingual educational materials are suggested.

Key words: COVID-19, dairy producer, health, business

\section{INTRODUCTION}

Since the emergence of the coronavirus disease 2019 (COVID-19) worldwide pandemic, caused by a severe acute respiratory syndrome coronavirus 2 (SARSCoV-2), significant global health, social, and economic disruptions have been observed. As of August 2021, the number of US patients who had succumbed to this 
infectious disease surpassed 500,000 (CDC, 2021). Furthermore, based on recent statistics from the Bureau of Economic Analysis from the US Department of Commerce (BEA, 2021), the pandemic resulted in a $3.5 \%$ deceleration of the US economy during 2020.

Dairies are labor-intensive production systems where cows must be fed, milked, and cared for $24 \mathrm{~h} / \mathrm{d}$ and 7 d/wk. In the United States, approximately $79 \%$ of the milk supply is produced on farms that employ immigrant labor, mostly of Latino ethnicity (Hadley et al., 2002; Adcock et al., 2015). Labor security has been a critical operating challenge in dairy production systems for approximately 2 decades (Hadley et al., 2002; Mugera and Bitsch, 2005); ensuring the health and safety of dairy workers during this pandemic is essential for the sustainability of this industry. Furthermore, disruptions in the foodservice supply chain and export markets have resulted in financial losses and cash-flow constraints for all agriculture sectors, including the dairy industry. For instance, dairy producers experienced an almost 50\% drop in milk prices at the beginning of the pandemic (CME, 2020). These drastic changes in income were reported as the most critical factor affecting dairy farmers early in the pandemic, causing anxiety and financial and mental stress (NMPF, 2020).

Understanding the concern of dairy producers regarding the health risk and effect on business of the pandemic, and the mitigation measures adopted, will inform and guide not only COVID-19 outreach efforts but also preparedness and response if future pandemics or uncertainties arise. Thus, the objective of the present study was to document dairy producers' perceptions and concerns regarding the effect of COVID-19 on the health of the workforce and business during the second wave of the pandemic.

\section{MATERIALS AND METHODS}

An anonymous survey tool was designed to collect information on how COVID-19 affected worker health and business of dairy producers at the beginning of the pandemic (Supplemental Table S1, https://data .mendeley.com/datasets/c6nbrv9nxc/1; Silva del Rio et al., 2021). The survey included a total of 14 questions; all questions were closed (multiple choice) except for 1 main question and 1 subquestion that were open-ended. Survey exemption was obtained from the Washington State University Institutional Review Board (Pullman, WA) before distribution. Four states (CA, ID, $\mathrm{SD}$, and WA) were chosen to deploy the survey based on researcher access to mailing list of licensed dairy producers (researchers held professional appointments on those states). The survey was mailed during the months of June and July (2020) to all licensed dairy producers in the aforementioned states and was accompanied with a monetary incentive only in the state of California (gift card). Responses received within 2 mo after mailing were included in the study. Researchers manually entered survey responses into Qualtrics XM (Provo, https://www.qualtrics.com/), and those were transferred into a spreadsheet for analysis (Excel 2010, Microsoft Corp.). Descriptive statistics were generated with SAS (version 9.4; SAS Institute Inc.) using the MEANS and FREQ procedures. Reported percentages were rounded to the nearest decimal point. Percentages for responses conditional to previous questions were calculated based on the answer to the previous question. In some cases, fewer answers were provided to the conditional compared with the initial question; in those cases, percentages do not add up to 100 .

Multiple correspondence analysis was performed using JMP 16 PRO (SAS Institute Inc.) to understand how respondent's dairy size [number of cows: small $(<100)$, medium (100-499), medium-large (500-1,999), or large $(\geq 2,000)$; this classification was a modification, based on our data characteristics, of the herd categories included in NAHMS (NAHMS-USDA, 2018)] and state (CA, ID, SD, or WA) related to answers to a selection of relevant questions from the survey. The questions selected for multiple correspondence analysis were as follows: (1) How concerned are you with COVID-19 being a risk to human health on your dairy? (very concerned, somewhat concerned, or not concerned at all); (2) Has anyone working on the dairy become sick with COVID-19? [yes (suspected and confirmed) or no]; (3) How concerned do you think your employees are about the health risks of COVID-19 on your dairy? (very concerned, somewhat concerned, or not concerned at all); (4) Have you provided your employees with any COVID-19 safety information or training? [yes (done and intended) or no]; (5) How concerned are you with COVID-19 having a negative impact on your dairy business? (very concerned, somewhat concerned, or not concerned at all); and (6) Do you have an emergency plan in place if a COVID-19 outbreak happens on your dairy? [yes (done and intended) or no]. Responses from the open-ended question, "Where did you get materials for the information/training?" were evaluated and grouped in the following categories: state agencies, dairy associations or milk processors, private industry, and universities or extension services. The accompanying figures were created using SigmaPlot (version 14.0; Systat Software Inc.) and JMP.

\section{RESULTS}

A total of 226 survey responses were received during June and July (2020). By state, response rates to the 
Table 1. Distribution of responses by state and dairy size and number of employees

\begin{tabular}{|c|c|c|c|c|c|c|c|}
\hline State & $\begin{array}{c}\text { Response } \\
{[\mathrm{n}(\%)]}\end{array}$ & \multicolumn{4}{|c|}{ Dairy size ${ }^{1}(\%)$} & \multicolumn{2}{|c|}{ Number of employees } \\
\hline California & $110(48.7)$ & 6.4 & 32.8 & 64.4 & 67.3 & 16 & 12 \\
\hline South Dakota & $49(21.7)$ & 58.1 & 26.2 & 11.0 & 9.1 & 10 & 14 \\
\hline Washington & 31 (13.7) & 12.9 & 26.2 & 6.8 & 10.9 & 10 & 13 \\
\hline
\end{tabular}

${ }^{1}$ Number of cows: small $(<100)$, medium (100-499), medium-large (500-1,999), or large $(\geq 2,000)$.

survey were as follows: $9.3 \%$ for California $(\mathrm{n}=110)$, $8.6 \%$ for Idaho $(\mathrm{n}=36), 31.4 \%$ for South Dakota $(\mathrm{n}=$ $49)$, and $10.0 \%$ for Washington $(\mathrm{n}=31)$. Overall, the distribution of responses received was by dairy size as follows: small (14.1\%), medium $(27.7 \%)$, medium-large $(33.2 \%)$, or large $(25.0 \%)$. The distribution of responses received by state was as follows: California (48.7\%), Idaho $(15.9 \%)$, South Dakota $(21.7 \%)$, or Washington $(13.7 \%)$. Further details on the distribution of responses by state and dairy size and dairy demographics are provided in Table 1. Twelve percent of the respondents reported that they had suspected $(3.3 \%)$ or confirmed (8.5\%) COVID-19 cases on their premises (n by dairy size: 2 small, 1 medium, 6 medium-large, 16 large; n by state: $13 \mathrm{CA}, 3 \mathrm{ID}, 5 \mathrm{SD}, 4 \mathrm{WA})$.

Most respondents were very concerned or somewhat concerned with COVID-19 being a risk to human health on their dairies $(75.0 \%)$. However, some respondents (25.0\%), especially those from small and medium dairies, expressed no concern with COVID-19 being a risk to human health on their dairies. Figure 1 represents the distribution of respondents' health concerns by dairy size (panel A) and state (panel B). Similarly, most respondents $(73.5 \%)$ perceived that their employees were very concerned or somewhat concerned about COVID-19, while $26.5 \%$ perceived that their employees were not worried at all about COVID-19. Figure 2 represents the distribution of respondents' perception of employees' concern by dairy size (panel A) and state (panel B). When asked about their main concern, respondents mentioned their families' health (75.6\%), employees' health (74.4\%), and employees' families' health $(65.9 \%)$.

Most respondents (91.5\%) reported that they had access to adequate information and resources to reduce the health risks of COVID-19 on their dairies. Nevertheless, $8.5 \%$ of the respondents encountered challenges acquiring adequate information due to feeling overwhelmed with too much information or not having enough time to search for information. Information about COVID-19 health risks and the coronavirus pandemic was obtained from online news sources and websites (47.3\%), television (35.8\%), family and friends
$(27.4 \%)$, radio $(17.3 \%)$, or social media $(16.8 \%)$. Some respondents $(24.8 \%)$ eventually became overwhelmed and stopped looking for information.

The majority of respondents reported that they had provided their employees with COVID-19 safety information or training (77.8\%). An additional 3.8\% of respondents planned to provide COVID-19 safety information and training, and $18.4 \%$ were not going to provide any information or training to their employees because, among other reasons, training was not considered necessary $(43.3 \%)$. A summary of responses to provided COVID-19 information or training, language, and topic of the provided information or training by dairy size and state is presented in Table 2. Participants $(67 \%)$ reported sources for training information that included the following: federal or state agencies (36.4\%), dairy associations or milk processors (18.5\%), private industry (i.e., human resources and safety consultants, and technical services; $33.1 \%$ ), and (or) universities or extension services $(3.8 \%)$.

The most commonly implemented mitigation measures for COVID-19 on the dairies were provision of hand sanitizer or disinfectant wipes $(78.8 \%)$ and frequent hand-washing instructions for employees (76.0\%). Additionally, the majority of respondents reported having implemented social distancing of at least 6 feet $(59.3 \%)$ and limited employees from congregating in groups $(53.8 \%)$. The use of masks and restriction of visitors on the dairy, however, were less popular (49.4 and $44.4 \%$, respectively). Responses to implemented mitigation measures by dairy size and state are summarized in Table 3.

Most of the respondents were very concerned or somewhat concerned about the effect of COVID-19 on business (92.2\%), whereas $7.8 \%$ were not concerned at all. Figure 3 represents the distribution of respondents' business concerns by dairy size (panel A) and state (panel B). Seventy-three percent of respondents indicated their business-related concerns associated with COVID-19 (Table 4). Some respondents $(29.2 \%)$ had an emergency plan in place if a COVID-19 outbreak happened on their premises, $24.0 \%$ were working on having one, and $46.8 \%$ did not have an emergency plan. 
Valldecabres et al.: DAIRY INDUSTRY TODAY

Table 2. Summary of responses to provided COVID-19 information or training by dairy size and state

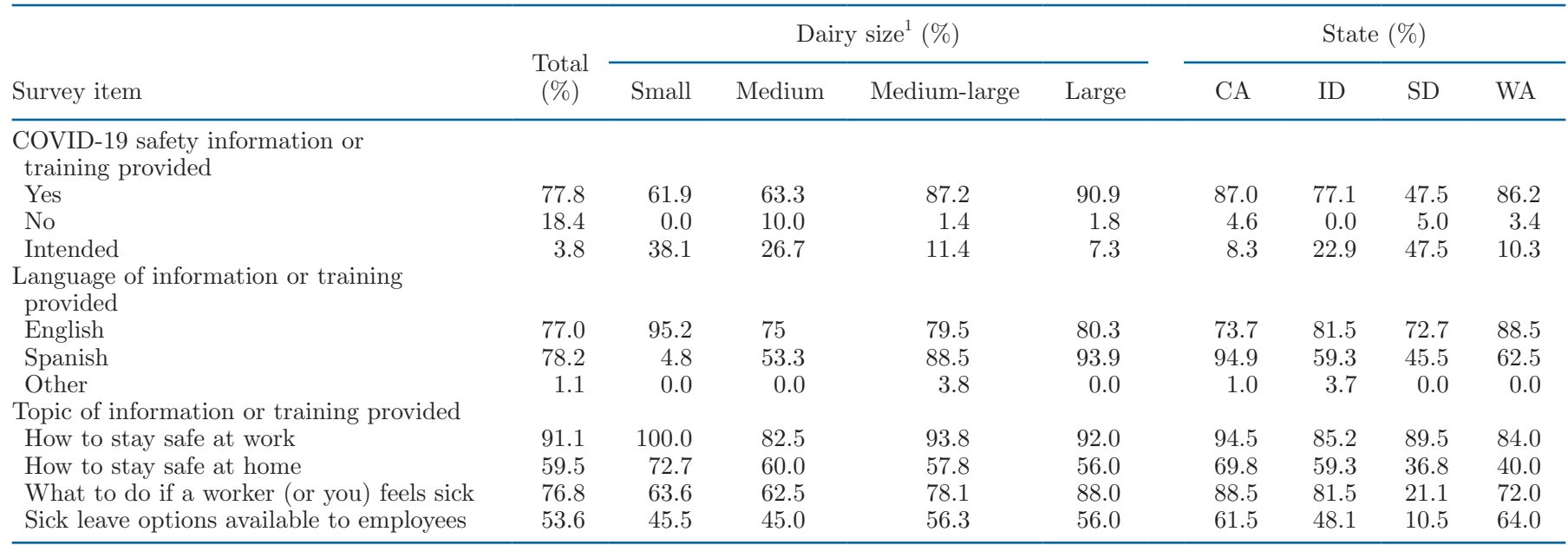

${ }^{1}$ Number of cows: small $(<100)$, medium (100-499), medium-large $(500-1,999)$, or large $(\geq 2,000)$.
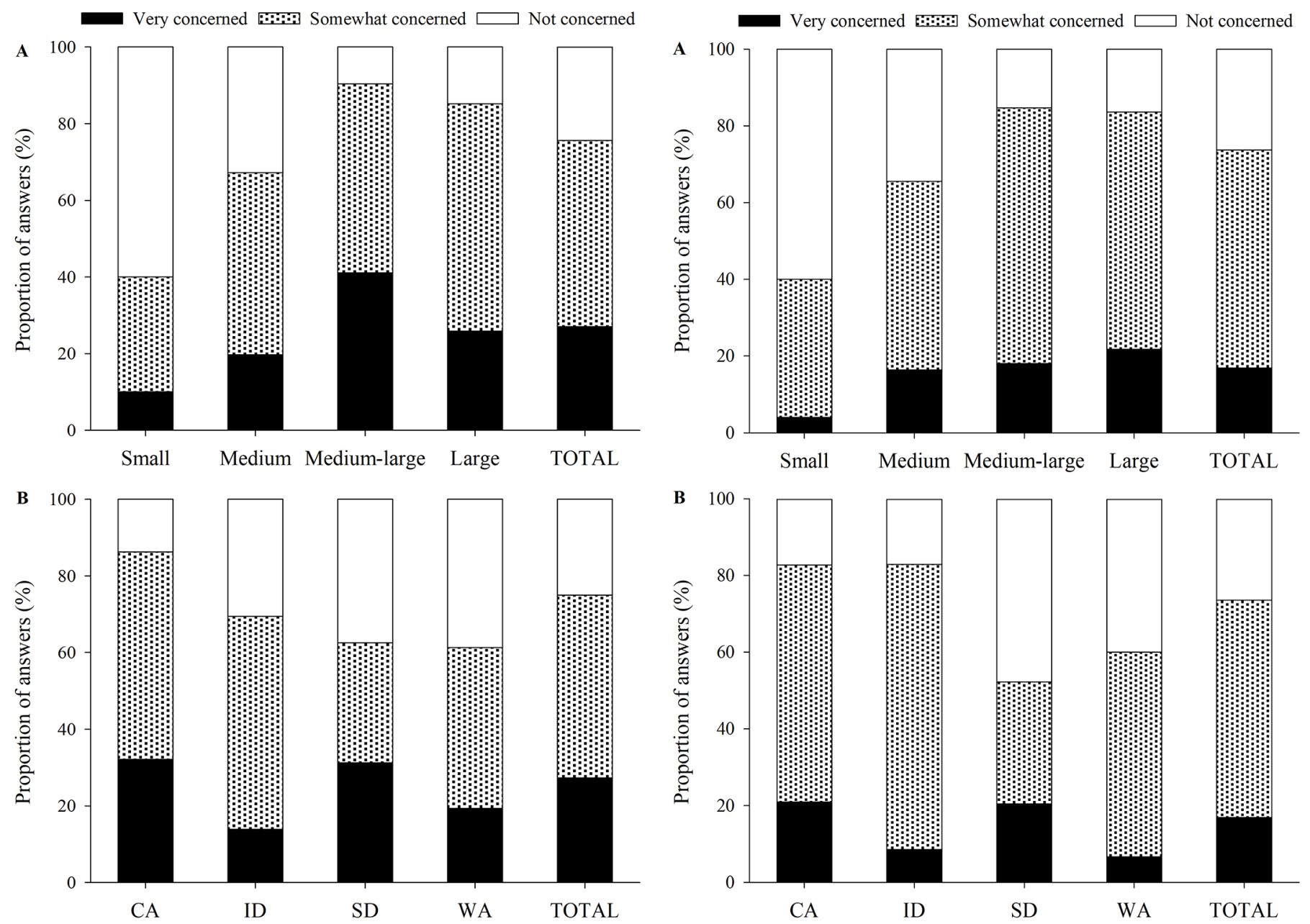

Figure 1. Answers to "How concerned are you with COVID-19 being a risk to human health on your dairy?" Panel A answers stratified by dairy size [(number of cows); small $(<100)$, medium (100-499), medium-large (500-1,999), large $(\geq 2,000)]$. Panel B answers stratified by state.

Figure 2. Answers to "How concerned do you think your employees are about the health risks of COVID-19 on your dairy?" Panel A answers stratified by dairy size [(number of cows); small $(<100)$, medium (100-499), medium-large (500-1,999), large $(\geq 2,000)]$. Panel $B$ answers stratified by state. 
Table 3. Summary of responses to implemented control measures for COVID-19 by dairy size and state

\begin{tabular}{|c|c|c|c|c|c|c|c|c|c|}
\hline Control measure for COVID-19 & $\begin{array}{l}\text { Total } \\
(\%)\end{array}$ & \multicolumn{4}{|c|}{ Dairy size ${ }^{1}(\%)$} & \multicolumn{4}{|c|}{ State $(\%)$} \\
\hline Provide and require employees to wear masks & 49.4 & 26.3 & 32.7 & 60.0 & 62.0 & 63.5 & 25.8 & 18.8 & 66.7 \\
\hline Provide hand sanitizer or disinfectant wipes & 77.8 & 47.6 & 74.5 & 85.5 & 89.8 & 88.0 & 81.8 & 50.0 & 82.8 \\
\hline $\begin{array}{l}\text { Limit employees from congregating in groups } \\
\text { (i.e., in break rooms) }\end{array}$ & 53.8 & 26.3 & 49.0 & 59.6 & 63.3 & 63.8 & 46.9 & 40.6 & 48.3 \\
\hline $\begin{array}{l}\text { Change workflow or scheduling to reduce } \\
\text { employee interaction }\end{array}$ & 17.4 & 9.1 & 18.4 & 15.4 & 23.4 & 18.5 & 10.3 & 23.5 & 14.3 \\
\hline $\begin{array}{l}\text { Restricted visitors on the dairy } \\
\text { (i.e., consultants, veterinarians, sales } \\
\text { representatives) }\end{array}$ & 44.4 & 26.1 & 38.0 & 50.9 & 51.0 & 50.6 & 41.9 & 30.6 & 46.4 \\
\hline
\end{tabular}

${ }^{1}$ Number of cows: small $(<100)$, medium $(100-499)$, medium-large $(500-1,999)$, or large $(\geq 2,000)$.

Responses to emergency plan questions by dairy size and state are summarized in Table 5. Among those with an emergency plan or an emergency plan in development, most ( $74.1 \%$ ) reported having access to adequate information and assistance to develop an appropriate emergency plan.

Multiple correspondence analysis is presented in Figure 4. There was an association between respondents from California, large, and medium-large dairies and reporting to have provided training to their employees, to have an emergency plan in place if an outbreak happened at their premises, and being very concerned about effect on business. An association was observed between respondents from small dairies and reporting not being concerned about COVID-19 being a risk to human health, perceiving that the employees were not worried about the health risks of COVID-19, and not having an emergency plan in place if an outbreak happened at their premises. Respondents from Idaho and Washington reported that they were somewhat concerned about COVID-19 being a risk to human health, perceived that the employees were somewhat concerned about the health risks of COVID-19, and that they were somewhat concerned about the effect on business of COVID-19. The first 2 dimensions of the analysis accounted for $57 \%$ of the total inertia (dimension 1: $36.3 \%$; dimension 2: $20.9 \%$ ).

\section{DISCUSSION}

The goal of our survey was to understand how dairy producers from California, Idaho, South Dakota, and Washington perceived the health and business implications of the COVID-19 pandemic as well as to describe the mitigation measures that were adopted. Our survey was limited to 4 states that represent over $30 \%$ of the cows and $7 \%$ of dairy producers in the United States.
Thus, caution is advised when extrapolating the results presented herein. Also, it should be noted that the focus of our survey was on dairy producers' perceptions; however, dairy managers and dairy workers might have had different perceptions of the health risk associated with the pandemic as well as their access to mitigation measurements and training. Our survey responses were received while the United States was undergoing the second wave of COVID-19 infections based on the statistics from the COVID-19 Dashboard by the Center for Systems Science and Engineering at Johns Hopkins University (Dong et al., 2020). However, the infection rates at the time of survey implementation differed among participant states; in California and Idaho, the new infection cases were above or approximately at the US average, whereas in WA and SD, they were below average. Thus, the urgency of the pandemic might have been perceived differently across states. Furthermore, the survey response rate was relatively low, and this might have introduced some bias in our results. It is plausible that mostly progressive and concerned dairy producers were among respondents. Our survey revealed that most dairy producers were somewhat or very concerned with the effect on business of the pandemic, but approximately a quarter of dairy producers reported lack of concern regarding the health risk associated with COVID-19. Furthermore, the reported adoption of mitigation measurements varied based on the type of measurement, the dairy size, and the state.

In our study, few dairies reported that they had confirmed or suspected COVID-19 cases on their premises. However, many dairy producers from Minnesota and Wisconsin reported COVID-19 cases in their facilities when interviewed in June and July of 2020 (Yung et al., 2021). The geographic differences between studies may have explained the disparity in the reported number of cases. It is also possible that Yung et al. (2021) were 
Table 4. Summary of reported concerns about effect of COVID-19 on the dairy

\begin{tabular}{|c|c|c|c|c|c|c|c|c|c|}
\hline Concern & $\begin{array}{c}\text { Total } \\
(\%)\end{array}$ & \multicolumn{4}{|c|}{ Dairy size $^{1}(\%)$} & \multicolumn{4}{|c|}{ State $(\%)$} \\
\hline Reduced availability of products and supplies & 22.0 & 21.1 & 26.7 & 27.6 & 43.5 & 24.0 & 21.2 & 16.1 & 24.1 \\
\hline $\begin{array}{l}\text { Reduced service provided by allied industry } \\
\text { personnel }\end{array}$ & 20.8 & 21.1 & 20.9 & 29.9 & 24.6 & 20.0 & 15.5 & 16.1 & 34.5 \\
\hline $\begin{array}{l}\text { Employees taking sick leave or not showing } \\
\text { up for work }\end{array}$ & 18.5 & 5.3 & 19.8 & 18.4 & 34.8 & 26.7 & 12.1 & 3.2 & 20.7 \\
\hline
\end{tabular}

${ }^{1}$ Number of cows: small $(<100)$, medium (100-499), medium-large (500-1,999), or large $(\geq 2,000)$.

able to get more accurate information through phone interviews. Nevertheless, based on the field experience of the authors, dairy producers may have been reluctant to report COVID-19 cases during the pandemic due to
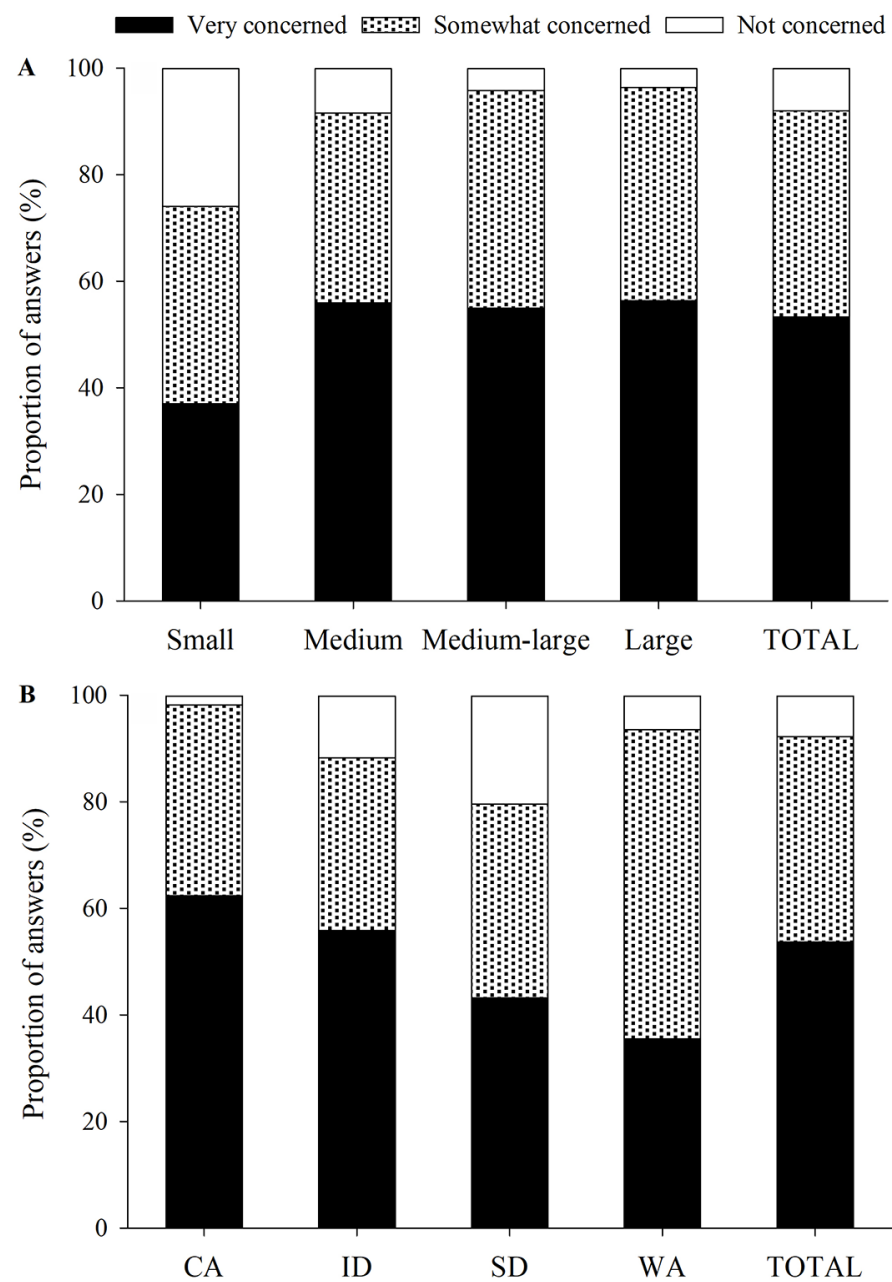

Figure 3. Answers to "How concerned are you that COVID-19 will have or has had a negative business impact on your dairy?" Panel A answers stratified by dairy size [(number of cows); small $(<100)$, medium (100-499), medium-large (500-1,999), large $(\geq 2,000)]$. Panel B answers stratified by state. possible county-level Human Health Services department intervention and labor shortage repercussions if all labor exposed had to be quarantined.

In agreement with our findings, Yung et al. (2021) reported that respondents were concerned about the safety of their families, their employees, and their employees' families. In our study, small dairies had low concerns about the health risk of COVID-19; their nondependency on external labor and limited social interactions outside the family most likely explained this observation. However, large dairies were concerned about the health risks of COVID-19. For decades, dairy operations have been facing labor security challenges (Mugera and Bitsch, 2005), and labor shortages could be disastrous in the event of an outbreak. Among states, in South Dakota, most respondents reported no concerns about COVID-19, which could also be explained by the relative low number of new infection cases reported during survey deployment or by $65 \%$ of the herds being small and medium size in that state (less dependency on external labor). Some of the respondents that showed a lack of concern about health implications of COVID-19 left comments expressing their disagreement with the political and social environment during the pandemic. It is noteworthy that although $25 \%$ of the respondents disclosed that they were very concerned about the health implications of COVID-19, only $17 \%$ perceived that their employees were also very concerned. These results aligned with the input provided by some producers in the open comment section, where some dairy producers expressed their disagreement with the Latino social lifestyle during lockdown (i.e., open Latino flea markets, frequent family gatherings). However, based on dairy workers' and managers' responses to a multistate online survey (Ferreira et al., 2021), they were concerned about the health risks of COVID-19. It is also of note that this ethnic group has been disproportionately affected by COVID-19 (Dorn et al., 2020).

In response to the pandemic, most respondents indicated that they trained or had the intention to provide 
Table 5. Summary of responses to having a COVID-19 outbreak emergency plan by dairy size and state

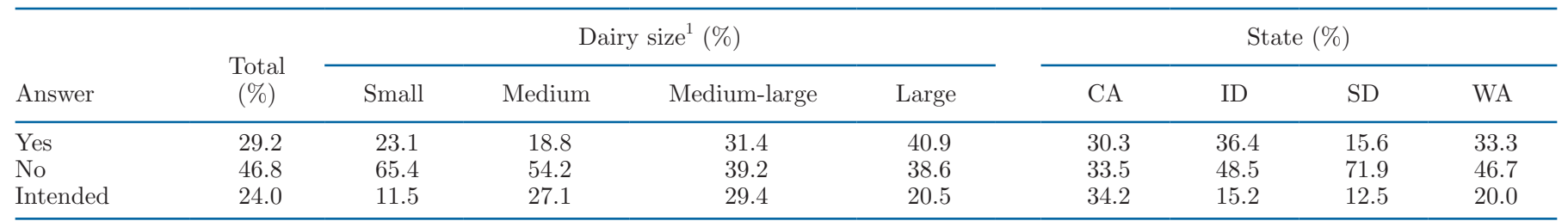

${ }^{1}$ Number of cows: small $(<100)$, medium (100-499), medium-large $(500-1,999)$, or large $(\geq 2,000)$.

safety training to their employees. Based on Ferreira et al. (2021), these trainings might have targeted mainly upper management positions (i.e., middle managers, area leaders). However, our survey was not designed to inquire about the extent and effectiveness of the trainings provided. Most dairies also stated that some level of biosecurity measures was implemented to mitigate the risk of a COVID-19 outbreak. Understanding the means and the language preferences to receive information are critical factors in designing effective biosecurity outreach curricula for dairy producers and employees. In our study, health agencies (especially the US Cen-

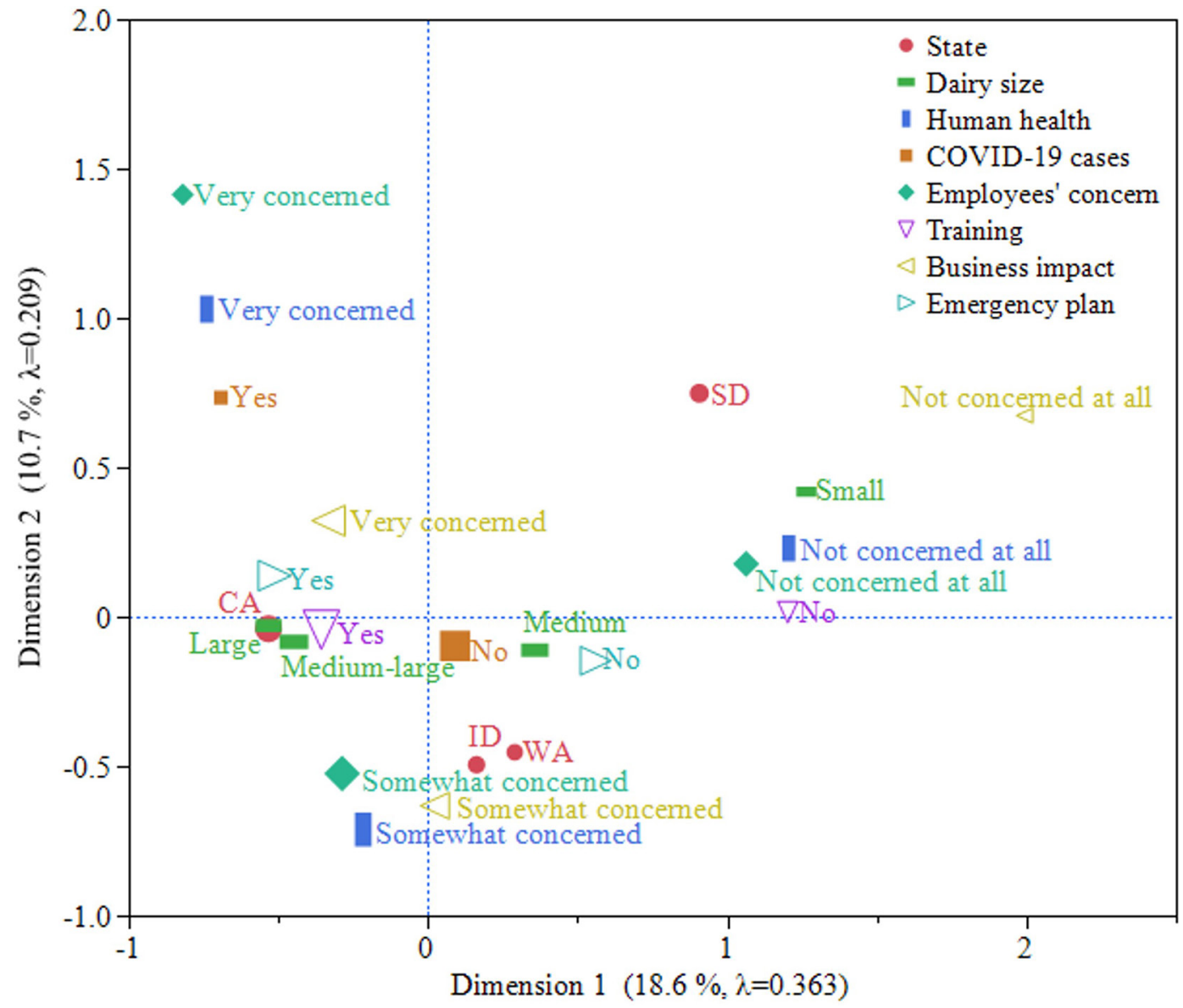

Figure 4. Map of multiple correspondence analysis evaluating the associations between state (CA, ID, SD, or WA) and dairy size (small: $<100$ cows; medium: 100-499 cows; medium-large: 500-1,999 cows; or large: $\geq 2,000$ cows) and answers to a selection of relevant questions as follows: (1) How concerned are you with COVID-19 being a risk to human health on your dairy? (very concerned, somewhat concerned, or not concerned at all); (2) Has anyone working on the dairy become sick with COVID-19? [yes (suspected and confirmed) or no]; (3) How concerned do you think your employees are about the health risks of COVID-19 on your dairy? (very concerned, somewhat concerned, or not concerned at all); (4) Have you provided your employees with any COVID-19 safety information or training? [yes (done and intended) or no]; (5) How concerned are you with COVID-19 having a negative impact on your dairy business? (very concerned, somewhat concerned, or not concerned at all); and (6) Do you have an emergency plan in place if a COVID-19 outbreak happens on your dairy? [yes (done and intended) or no]. The size of each symbol in the plot is proportional to the number of observations for each variable represented. 
ters for Disease Control), private consultants (mostly those specialized in safety), milk processing plants, and local and nationwide dairy associations were trusted sources for dairy producers when looking for health impact and training information. Yung et al. (2021) observed that $20 \%$ of dairy producers used local and national dairy associations as sources of information, and they argue that a more organized leadership from dairy associations will benefit preparedness efforts in future pandemics. Overall, based on our survey, information technologies (online resources) and traditional outlets (television news) were equally important means to obtain COVID-19 information. Social media was also a common resource to obtain health-impact related information in our study; based on the multistate online survey by Ferreira et al. (2021), social media had a larger popularity among dairy workers than upper management positions. Considering that $51 \%$ of all dairy labor is immigrant, and mostly Latino (Adcock et al., 2015), in the United States, it is not surprising that in our study, most producers from medium-large and large dairies reported having provided or intend to offer bilingual trainings to their workforce. Our results also showed that some respondents were not planning to provide training to their employees; these respondents were mainly from medium dairies in Idaho and Washington.

Based on dairy producers, the 2 most common measures implemented were instructing employees to wash hands frequently and providing hand sanitizer. These measures align with the US Centers for Disease Control recommendations at the beginning of the pandemic and the implemented measures reported by Yung et al. (2021). It is unclear if these measures were standard practices before the pandemic and were just re-emphasized at this time. Face covering, a control measure first proposed by the US Centers for Disease Control on April 3, 2020, was only popular on medium-large and large dairies. Yung et al. (2021) reported that $40 \%$ of dairy farms from Minnesota and Wisconsin implemented a face covering requirement, but only $14 \%$ enforced it. Likewise, $38 \%$ of dairy managers and workers reported that face coverings were required at work (Ferreira et al., 2021). Based on the authors' field observations in their home states (CA, ID, SD, and WA), face coverings on dairies were enforced only occasionally. Arguments used against it were reported by Yung et al. (2021) and included questioning their need in well-ventilated spaces and the discomfort in hot and humid weather. Social distancing, limiting employee gathering, and restricting visitors were biosecurity measures reported by most dairy producers, especially in medium-large and large dairies. Very few dairies adopted workflow or scheduling modifications as a mitigation strategy.
Most respondents expressed their concern about the business implications of the pandemic; large-dairy farmers were especially preoccupied with the lack of inputs and labor. These concerns were justified after the unprecedented restrictions on movements of people and goods during the pandemic. Furthermore, after the stay-at-home mandates forced restaurants and schools to close, some dairy producers were asked to dump milk as a short-term solution for the decline in milk demands. Twenty percent of the respondents were concerned about dumping milk, and this proportion was similar across different dairy sizes; however, $49 \%$ of respondents from Idaho reported being concerned about dumping milk. The vast majority of Idaho milk is purchased in bulk by the food service industry, which was significantly affected by the pandemic. Also, Idaho is not part of any federal milk marketing order and relies less heavily on cooperatives. All of these factors combined may explain the higher concern about having to dump milk for respondents from this state.

\section{CONCLUSIONS}

Our multistate survey was conducted during the early months of the pandemic. Most dairy producers were navigating business uncertainties while implementing measures to ensure the safety and health of their workforce, although most of our respondents indicated adequate availability of training materials. The secondary role of dairy industry service and education providers as sources of information suggest a need for future centralized efforts from dairy-specific organizations. Given the trajectory of the global pandemic, a re-assessment of the logistic, health, and business challenges faced during the pandemic is warranted to inform agencies involved in response to future pandemics.

\section{ACKNOWLEDGMENTS}

The authors appreciate the collaboration of all the US producers who kindly responded to our survey. We extend our appreciation to Marcelo Abreu (Veterinary Medicine Teaching and Research Center, Tulare, CA). The authors have not stated any conflicts of interest.

\section{REFERENCES}

Adcock, F., D. Anderson, and P. Rosson. 2015. The economic impacts of immigrant labor on U.S. dairy farms. Center for North American Studies. Accessed Aug. 19, 2020. https://www.nmpf.org/wp -content/uploads//immigration-survey-090915.pdf.

Bureau of Economic Analysis from the US Department of Commerce (BEA). 2021. News at a glance. Accessed May 24, 2021. https:// www.bea.gov/news/glance. 
Centers for Disease Control and Prevention (CDC). 2021. Weekly updates by select demographic and geographic characteristics. Accessed May 29, 2021. https://www.cdc.gov/nchs/nvss/vsrr/covid _weekly/index.htm.

Chicago Mercantile Exchange (CME). 2020. Dairy Market Outlook Q1 2020. Accessed May 22, 2020. https://www.cmegroup.com/ education/featured-reports/dairy-market-update-q1-2020.html.

Dong, E., H. Du, and L. Gardner. 2020. An interactive web-based dashboard to track COVID-19 in real time. Lancet Infect. Dis. 20:533-534. https://doi.org/10.1016/S1473-3099(20)30120-1.

Dorn, A. V., R. E. Cooney, and M. L. Sabin. 2020. COVID-19 exacerbating inequalities in the US. Lancet 395:1243-1244. https://doi .org/10.1016/S0140-6736(20)30893-X.

Ferreira, F. C., M. Rovai, N. Silva-del-Rio, M. Chahine, M. de Haro Marti, J. Wenz, and J. Dalton. 2021. Perspectives of dairy employees on the COVID-19 pandemic. J. Dairy Sci. 104(Suppl. 1):90. (Abstr.)

Hadley, G. L., S. B. Harsh, and C. A. Wolf. 2002. Managerial and financial implications of major dairy farm expansion in Michigan and Wisconsin. J. Dairy Sci. 85:2053-2064. https://doi.org/10 .3168/jds.S0022-0302(02)74283-5.

Mugera, A. W., and V. Bitsch. 2005. Managing labor on dairy farms: A resource-based perspective with evidence from case studies. Int. Food Agribus. Manag. Rev. 8:79-98. https://doi.org/10.22004/ag .econ.8140.

National Animal Health Monitoring System-United States Department of Agriculture (NAHMS-USDA). 2018. Accessed Sep. 14,
2020. https://www.aphis.usda.gov/animal_health/nahms/dairy/ downloads/dairy14/Dairy14_dr_PartIII.pdf.

National Milk Producers Federation (NMPF). 2020. A threat to dairy livelihoods. Accessed Jun. 2, 2020. https://www.nmpf.org/ coronavirus/.

Silva del Rio, N., M. Rovai, J. Wenz, J. Dalton, F. Ferreira, M. De Haro Marti, and M. Chahine. 2021. Survey: COVID-19 related information from dairy producers. Mendeley Data, V1, https://doi .org/10.17632/c6nbrv9nxc.1.

Yung, M. T., R. I. C. Vázquez, A. Liebman, A. Brihn, A. Olson, D. Loken, A. Contreras-Smith, J. Bender, and J. D. Kirsch. 2021. COVID-19 awareness and preparedness of Minnesota and Wisconsin dairy farms. J. Agromedicine https://doi.org/10.1080/1059924X .2021.1927925.

\section{ORCIDS}

A. Valldecabres $\odot$ https://orcid.org/0000-0002-3235-2487

F. C. Ferreira ๑ https://orcid.org/0000-0003-2387-2269

M. Chahine ํㅏ https://orcid.org/0000-0003-4434-5249

J. Dalton (1) https://orcid.org/0000-0003-4397-6647

M. de Haro Marti @ https://orcid.org/0000-0002-6750-3695

M. Rovai $\odot$ https://orcid.org/0000-0001-9201-1505

N. Silva-del-Río ๑ https://orcid.org/0000-0002-2826-6797 